\title{
Ultrasound assessment of the lateral collateral ligamentous complex of the elbow: imaging aspects in cadavers and normal volunteers
}

\author{
Pedro Augusto Gondim Teixeira - Patrick Omoumi • \\ Debra J. Trudell • Samuel R. Ward • Sophie Lecocq • \\ Alain Blum • Donald L. Resnick
}

Received: 15 November 2010 /Revised: 30 December 2010/Accepted: 13 January 2011 /Published online: 13 February 2011

(C) The Author(s) 2011. This article is published with open access at Springerlink.com

\begin{abstract}
Objective The Lateral Collateral Ligamentous complex (LCL) is an important stabiliser of the elbow. It has a Yshaped structure with three components. In this study, we sought to describe the ultrasound aspect of the individual components of this ligamentous complex and to evaluate the performance of ultrasound in both cadavers and in normal subjects.

Methods Ten cadaveric elbow specimens underwent highfrequency ultrasound. Two specimens were sliced and two were dissected for anatomical correlation. Ten elbows of normal subjects were also evaluated by ultrasound. The findings were compared.

Results The three components of the LCL could be visualised in all specimens and normal subjects with the exception of the proximal portion of one specimen. In $80 \%$
\end{abstract}

D. J. Trudell • S. R. Ward · D. L. Resnick

Department of Radiology,

Veterans Administration Medical Center,

3350 La Jolla Village Dr,

San Diego, CA 92161, USA

P. Omoumi

Department of Radiology,

Cliniques Universitaires St Luc-UC Louvain,

Brussels, Belgium

S. Lecocq · A. Blum

Service D'imagerie Guilloz, CHU Hôpital Central,

54000 Nancy, France

P. A. Gondim Teixeira $(\bowtie)$

Service d'Imagerie Guilloz, CHU Hôpital Central,

10 Boulevard Recteur Senn, appt 220,

54000 Nancy, France

e-mail: ped_gt@hotmail.com of the specimens and $100 \%$ of the healthy volunteers the proximal portion of the LCL could be separated from the extensor tendons.

Conclusion High-resolution ultrasound can assess all components of the LCL of the elbow and can distinguish them from surrounding structures.

Keywords Elbow · Lateral collateral ligament · Joint instability · Ultrasound · Anatomy

\section{Introduction}

The Lateral Collateral Ligamentous complex (LCL) of the elbow is traditionally described as a Y-shaped complex that has three components: the radial collateral ligament (RCL) extending from the lateral epicondyle to the annular ligament; the lateral ulnar collateral ligament (LUCL) extending from the lateral epicondyle to the supinator crest of the ulna; and the annular ligament, extending from the anterior margin of the ulna at the sigmoid notch to the supinator crest at the posterior margin of this bone, forming a ring that surrounds the radial head and neck. The proximal portion of the LCL, located above the annular ligament is composed of fibres of the RCL and the proximal fibres of the LUCL [1-3] (Fig. 1).

All components of this ligamentous complex function together as lateral stabilisers of the elbow joint and are the main constraints to posterolateral rotatory instability (PLRI) $[1,4]$. PLRI is characterised by various degrees of posterior dislocation of the radial head when the elbow is exposed to an association of axial compression, supination and valgus stress. This condition is usually chronic and is seen in patients with ligament laxity, overuse or previous trauma 


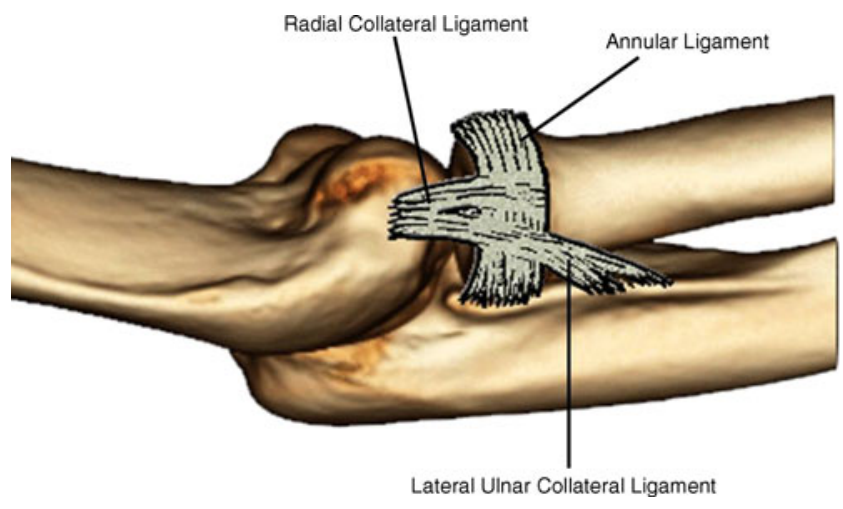

Fig. 1 Schematic representation of the lateral collateral ligamentous complex of the elbow

(fall on an outstretched hand). It may also be iatrogenic as some surgical approaches used to treat tennis elbow may jeopardise the LCL $[5,6]$. If untreated PLRI may lead to chronic disability, pain, degenerative joint disease, and may even progress to full dislocation of the elbow [5, 7]. Surgery may be indicated in cases of failure of the conservative measures [8]. Physical examination of this condition can be hindered by the patient guarding and sedation might be necessary [9]. Imaging can assist in the diagnosis of this condition and can differentiate it from an alternative diagnosis such as valgus elbow instability and complicated lateral epicondylitis which may have a similar clinical presentation [4].

Magnetic resonance imaging can be used as a noninvasive imaging technique to evaluate lesions of the LCL. This method allows, to some degree, the identification of its three components and it can be used in the setting of PLRI [5]. MR arthrography and intermediateweighted sequences have the highest sensitivity for the diagnosis of LUCL tears [10]. However, MR imaging of the LUCL can be difficult in the straight coronal plane due to its oblique course $[1,6,9]$. Images acquired using $3-\mathrm{D}$ sequences and in an oblique coronal plane adapted to the anatomy of the LUCL may help in analysing this ligament $[10,11]$. Nonetheless, Terada et al. was able to clearly visualise the LUCL in only $50 \%$ of cases in a series of 20 asymptomatic persons using an adapted protocol with oblique coronals [12].

Ultrasound is a method of choice for the study of some ligaments, providing high-resolution imaging with state-ofthe-art technology [13]. This method is widely available, has a lower cost compared with MR imaging, and is routinely performed for the diagnosis of refractory cases of lateral elbow pain. The ultrasound evaluation of the LCL has been reported [13-16]. However, none of these studies has focused on the individualisation of the components of the LCL, its bony attachments or its differentiation from surrounding structures with ultrasound.
In this study, we sought to evaluate the performance of ultrasound in the assessment of the LCL, focusing on the characterisation of the RCL, LUCL and annular ligament.

\section{Materials and methods}

Ten human elbow joints were obtained from 7 fresh frozen cadavers (6 males and 1 female, age at death: 58-92 years old). The specimens consisted of the wrist, forearm, elbow joint and proximal half of the arm. The specimens were deep-frozen at $-40^{\circ} \mathrm{C}$ for at least 3 days (Forma BioFreezer; Forma Scientific, Marietta, OH, USA). All specimens were allowed to thaw for $24 \mathrm{~h}$ at room temperature before ultrasound imaging.

Ultrasound of the cadaveric specimens was performed using an IU22 system (Philips, Best, the Netherlands) and a high-frequency linear transducer $(15-17 \mathrm{MHz})$. The actual examination frequency was $17 \mathrm{MHz}$ and the THI was not toggled. The same ultrasound device and probe that were used in the imaging of the whole specimens were used for the staged dissection.

We then evaluated ten elbows from 5 healthy volunteers (4 female and 1 male, age: 24-29 years old) with no history of previous trauma to the elbow and no abnormality at the physical examination. The same ultrasound technique and the same criteria adopted for the evaluation of the cadavers were used. Volunteer ultrasound was performed using an Aplio XG, SSA-790A Toshiba (Zilverstraat 1. 2718 RP, Zoetermeer, Netherlands) with an $8-15 \mathrm{Mhz}$ frequency linear transducer. The actual examination frequency was $12 \mathrm{MHz}$ and the 2-D THI was used.

Since this study was performed on donated cadavers, without any clinical information and asymptomatic volunteers, no ethics committee approval was needed at our institution.

Two fellowship-trained musculoskeletal radiologists (P. O. and P.G.T.), with 2 years of experience in musculoskeletal ultrasound, performed all the examinations and evaluated the images in consensus. All structures were examined in both the long axis and short axis directions. The elbows were examined in extension and in flexion (the LCL was taut and less prone to anisotropy artefacts in this position). The RCL, LUCL and annular ligament were evaluated at their attachments and along their length. The bony surface of the lateral epicondyle was also evaluated.

A ligament with continuous fibres and a preserved fibrillary pattern was considered to be normal at the ultrasound examination. The echogenicity of the ligament components was characterised as hypo- or hyperechogenic using the overlying muscles for comparison. The detectability (i.e. ease of detection) of all regions of the ligamentous complex and its differentiation from the 
overlying common extensor tendons was classified as good, poor or non-assessable. The detectability of the ligament was considered to be good when it could be clearly separated from the extensor tendons based on the expected site of insertion, fibre echogenicity and fibre orientation. The detectability was considered to be poor when the margins of these ligaments were indistinct and difficult to differentiate from the overlying extensor tendons, but a hypoechogenic band was still visualised in the expected location of the ligament. The ligament was considered as non-assessable when its fibres were not discernable at the ultrasound evaluation. At the attachment of the LCL at the supinator crest, the type of attachment of the annular and LUCL components was also assessed (separated versus conjoined attachment).

Immediately after imaging, the specimens were frozen again at $-40^{\circ} \mathrm{C}$ for at least three days. Two specimens were then sliced with a band saw into 3-mm thick slices in the coronal plane with fluoroscopic guidance. Photographs and radiographs (Faxitron HP 43805 N X-Ray System, HewlettPackard, Palo Alto, CA, USA; tube current, $30 \mathrm{kV}$; exposition time, $30 \mathrm{~s}$ ) of all sections were obtained. Two other specimens were dissected by an anatomist (S.W.) and photographs were taken during all the steps of the procedure. The progressive dissection technique described by De Maeseneer et al. was used [17]. In this technique the specimen undergoes imaging before and after the removal of each layer of tissue (e.g. dermis and hypodermis, periarticular muscles and capsulo-ligamentary structures). The ultrasound probe was wrapped with a latex glove to avoid contamination and all measures to prevent skin contact with the specimens were taken.

\section{Results}

The three components of the LCL could be visualised in all specimens and normal volunteers with the exception of the proximal portion of one specimen, which was not assessable due to a large calcification, located in the overlying extensor tendons. The medial attachment of the annular ligament on the sigmoid notch was not assessable in both cadaveric specimens and normal volunteers. The posterior attachment of the annular and the ulnar components on the supinator crest was visible in all cases.

The normal ultrasound aspect of each of the portions of the LCL of the elbow is depicted on Fig. 2.

The detectability of all the assessable components of the LCL was considered to be good in all normal volunteers. In one specimen the detectability of both the proximal portion of the LCL and the annular ligament was considered to be poor. The detectability of the LUCL was considered to be good in all the specimens.
The proximal LCL appeared hyperechogenic in all normal volunteers and in $50 \%$ of the cadaveric specimens.

The body of the annular ligament was visualised as a hypoechogenic band of tissue indistinguishable from the radial head cartilage in both cadavers and volunteers. Some hyperechogenic areas were seen eventually with the mobilisation of the probe, secondary to anisotropy artefacts due to the curved anatomy of this structure. The LUCL appeared hypoechogenic in both cadavers and volunteers.

At the proximal portion of the LCL, the RCL and LUCL could not be separated with ultrasound and a fibrillary pattern could be identified (Fig. 2a). These findings were similar in both cadaveric specimens and normal volunteers. The different components of the LCL were not separable at the level of the annular ligaments as their fibres blended with the latter. As the fibres of the LCL curved over the annular ligament a hypoechogenic zone of a few millimetres could be seen in some cadaveric specimens. In that area the fibrillary pattern could no longer be identified, and evaluation of the LCL was difficult. This hypoechogenic zone was not seen in the volunteers. Ultrasound imaging with the elbow in a flexed position ameliorated the visualisation of the proximal LCL in cadavers by reducing the size of the hypoechogenic zone described. The degree of flexion did not influence the ultrasound LCL aspect in normal volunteers.

The attachment of the annular ligament appeared as a thin hypoechogenic band of tissue. A triangular hyperechogenic area could be seen between the ligament's insertion and the radial head (Figs. $2 b$ and 3 ). The attachment of the annular ligament was distinct from the attachment of the LUCL in $52 \%$ and it was continuous to it in $48 \%$ of the elbows evaluated.

The distal portion of the LCL is composed mainly of the LUCL fibres. This ligament fanned out close to its insertion and was easily separated from the periligamentous soft tissues, which consisted mainly of hyperechogenic fatty tissue (Figs. 2c and 4).

Differentiation of the proximal portion of the LCL from the extensor tendons was possible in $80 \%$ of the specimens and in $100 \%$ of the controls, by the detection of a hyperechogenic line separating the two structures. This differentiation was easier in the normal volunteers (Fig. 5).

We identified a small bony tubercle in the anterior-most part of the lateral humeral epicondyle that was located at the site where the hyperechogenic line described between the LCL and the extensor tendons reaches the epicondyle (Fig. 6). This structure was found in $61.1 \%$ of the lateral epicondyles analysed (62\% of the cadaveric specimens and in $60 \%$ of the volunteers).

All the components of the LCL were identified in the gross anatomical studies. In the dissection the components of the LCL were repetitively and consistently identified 


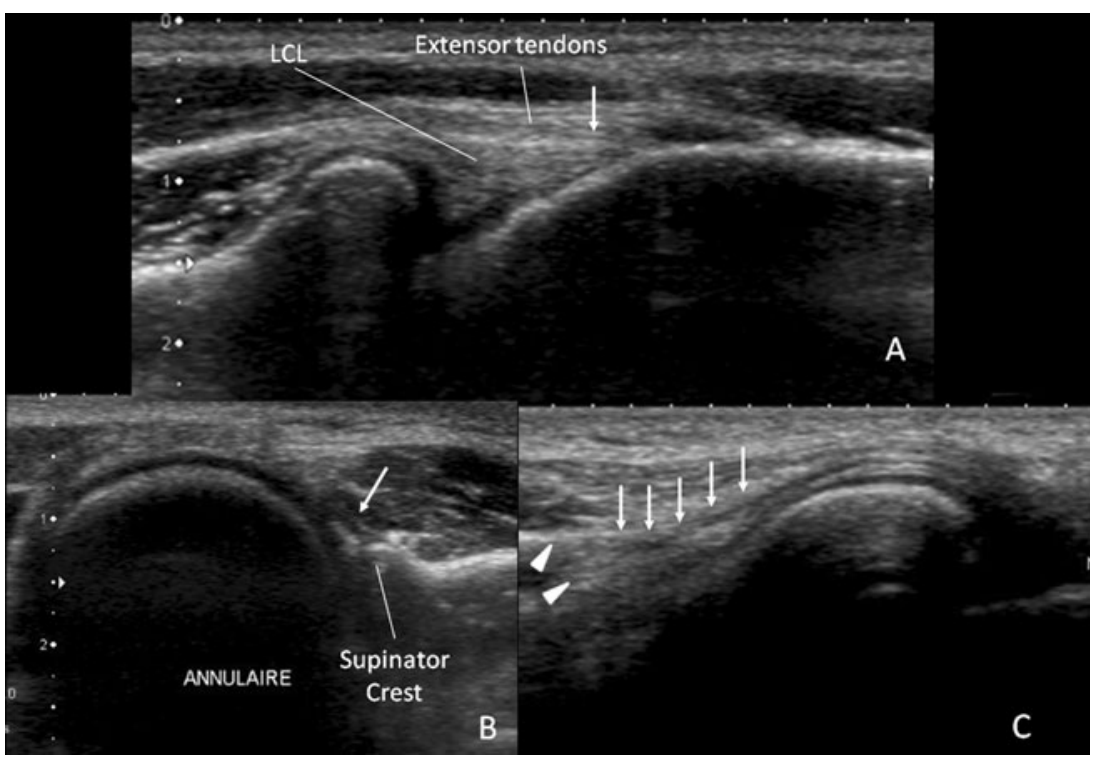

Fig. 2 a Long axis ultrasound image of the anterolateral surface of the extended elbow at the level of the lateral humeral epicondyle demonstrating a clear separation of the proximal portion of the LCL from the overlying extensor tendons and the echogenic line between these two structures (arrow) in a 25 -year-old female control. b Short axis ultrasound image at postero-lateral elbow at the level of the radial head of 29-year-old male volunteer also showing the annular ligament

throughout the stages. In the last stage of the dissection, direct ultrasound evaluation of these ligaments was performed. The ligaments were then removed and ultrasound followed to confirm their absence. This indicates that the structures visualised at ultrasound were indeed the components of the LCL. The specimen sectioning was consistent and confirmed the anatomical features of the LCL described in the literature. The sectioning also confirmed the

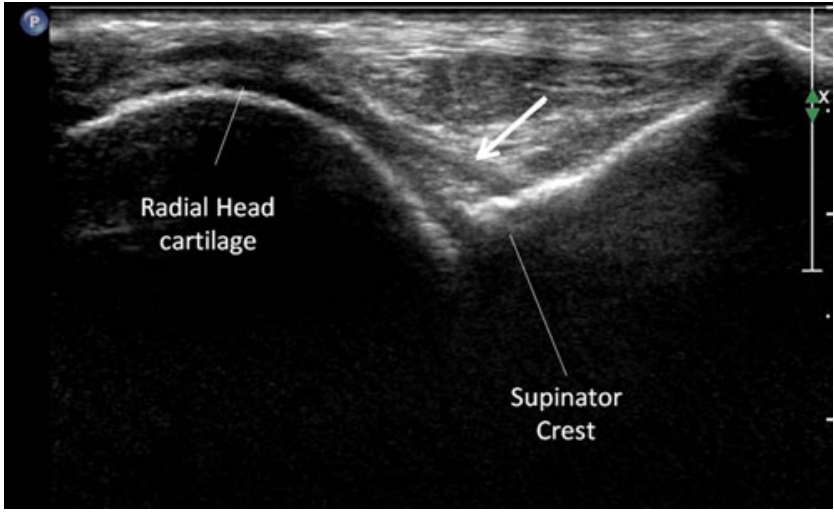

Fig. 3 Short axis ultrasound image at posterolateral elbow at the level of the radial head showing the posterior attachment of the annular ligament to the supinator crest in an 81-year-old female cadaver specimen. The superficial fibres of the annular ligament are seen as a hypoechoic band of tissue (arrow). Deep to this structure a triangular region of hyperechogenic fatty tissue is shown. The deep fibres of the annular ligament attachment could not be differentiated from the radial head cartilage and its posterior attachment (arrow) at the supinator's crest. c Oblique long axis image of the posterolateral elbow at the level of the radial head of the same subject demonstrating the LUCL and its insertion (arrows) to the supinator tubercle of the ulna (arrowheads). Note the hyperechogenicity of the surrounding soft tissues facilitating the identification of this structure

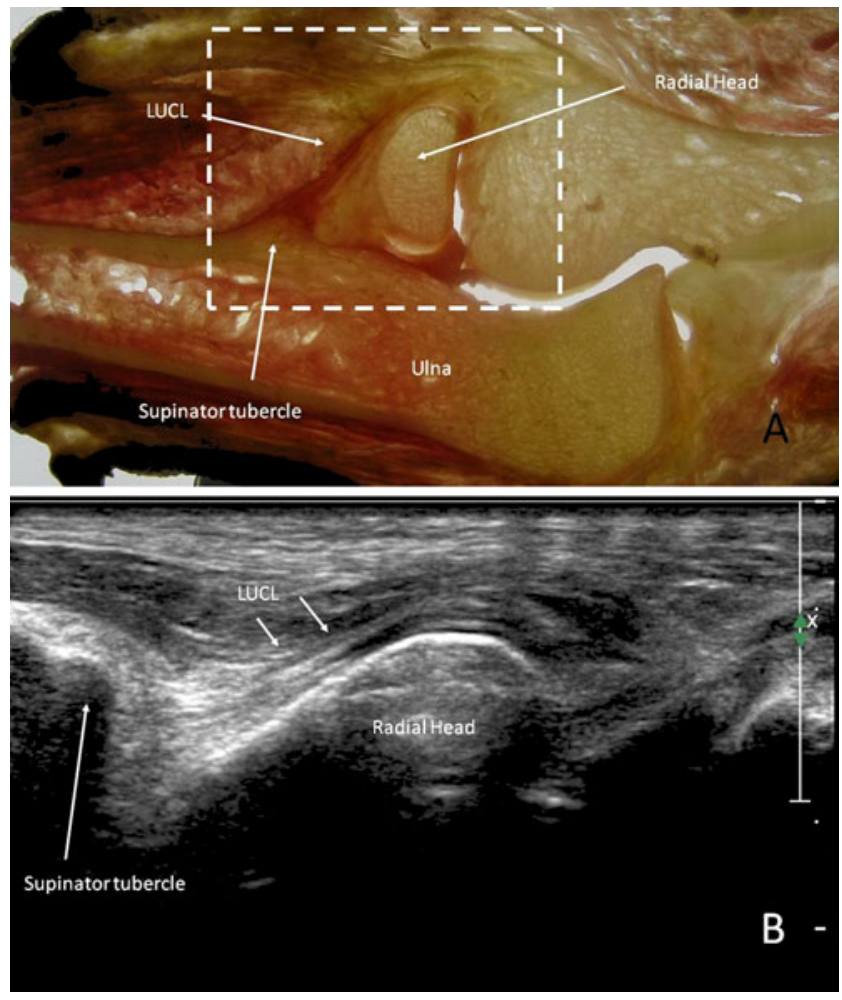

Fig. 4 A) Coronal section of the elbow at the level of the lateral ulnar collateral ligament. The white dotted square marks the corresponding area of the ultrasound image on $\mathrm{B}$ acquired in an oblique long axis direction at the posterolateral elbow at the level of the radial head 

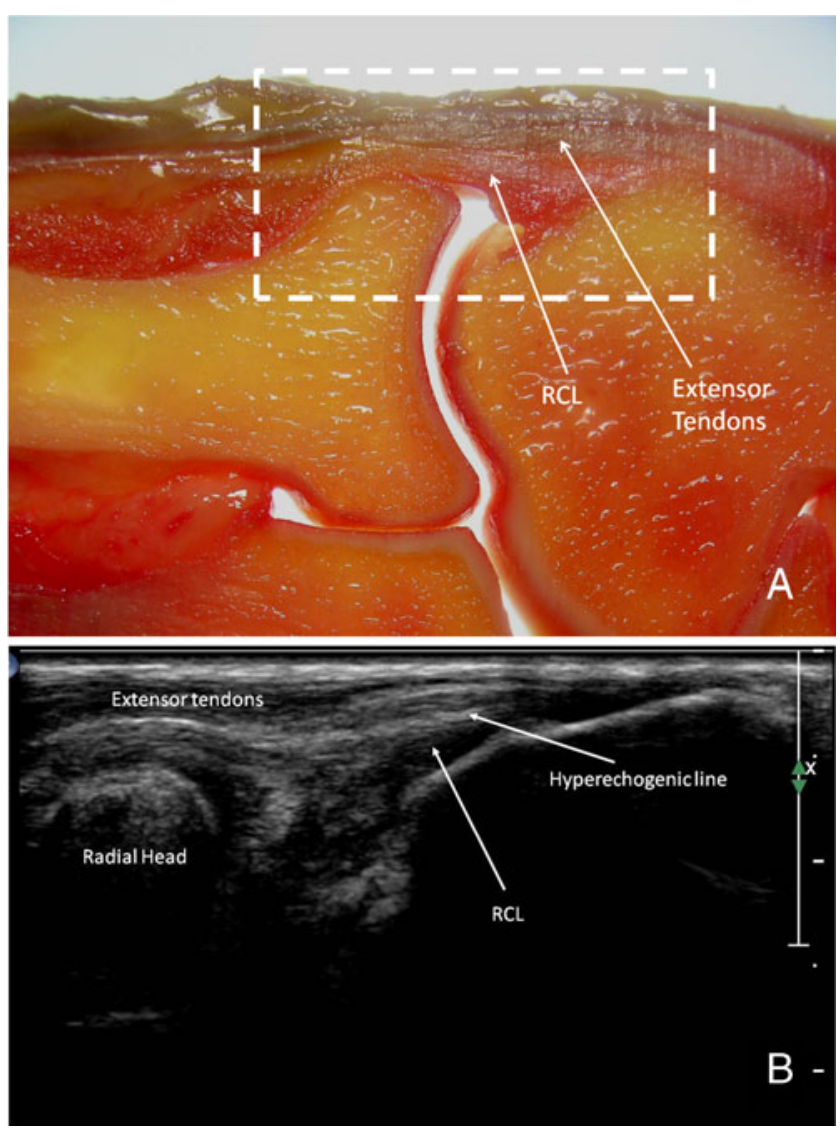

Fig. 5 A) Coronal section of the elbow at the level of the radial collateral ligament. The white dotted square marks the corresponding area of the ultrasound image on B acquired in the long axis direction at the anterolateral surface of the elbow

ultrasound features that help to differentiate the LCL from the overlying tendons (e.g. the echogenic line and the bone tubercle).

The authors felt no need to perform anatomical correlation in all the specimens because the anatomy of the region has already been fully described and the ultrasound findings were repeatedly confirmed in the specimens we did dissect and section [1, 18-22]. We still consider the data of specimens without anatomical correlation useful, because the information helps to characterise the ultrasound features of the LCL.

\section{Discussion}

The LUCL is classically described as the main constraint to PLRI, and hence the most important component of the LCL [1]. However, more recent anatomical and biomechanical studies have challenged that concept and the entire LCL is now considered by many to be equally important for lateral elbow stability $[2,18,23-28]$. Although the anatomy of the LCL has been well described, only a few studies have focused on its ultrasound aspects [16, 29-32]. The ultrasound appearance of LUCL and the annular ligament has been described in the literature, but limited information is available on the relations between the components of the LCL and the differentiation of these components from the nearby tendons $[16,29,30]$.

An association of LCL injury and lateral epicondylitis has been reported. When LCL insufficiency is present, there is a higher probability of conservative treatment failure [9, 33]. Thus, the LCL should be assessed systematically when evaluating lateral epicondylitis [13]. Tears of the LCL have to be diagnosed pre-operatively. If there is an LCL injury the loss of the secondary lateral stabilisers secondary to extensor tendon release surgery may lead to post-operative PLRI $[9,13]$.

The proximal part of the LCL including its attachment to the lateral epicondyle is the most common site of tears. McKee et al., in a series of 62 dislocated elbows, also described midsubstance tears and avulsions at the ulnar
Fig. 6 Demonstration of the variations of the osseous surface of the lateral epicondyle in a 58-year-old male specimen. a High-definition radiography demonstrating the anterior tubercle (arrowhead), note the radiolucent line between the tendons and the proximal part of the LCL. b Corresponding long axis anterolateral ultrasound image of the elbow at the level of the lateral epicondyle demonstrating the anterior tubercle (arrowhead). Note that the radiolucent line in (A) appears hyperechogenic on ultrasound
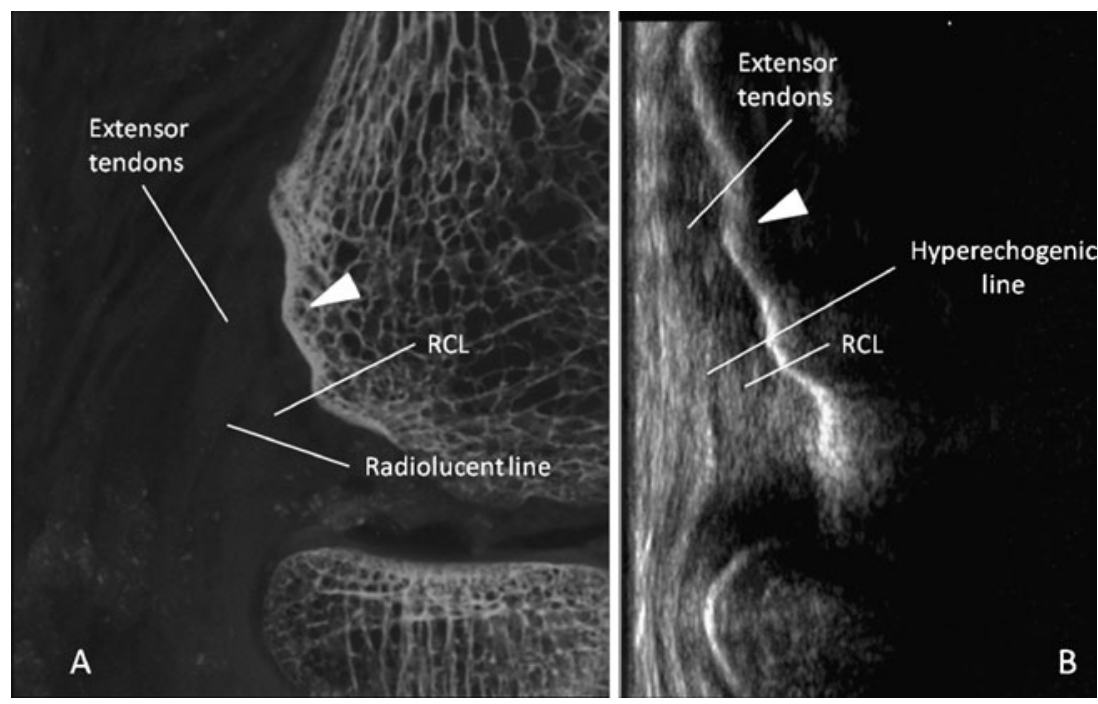
attachment of the LCL [18]. These types of injuries, although less frequent, warrant the need for a thorough analysis of each of the individual components of the LCL.

There have been previous reports of the ultrasound evaluation of the annular ligament in the case of pulled elbow (Nursemaid elbow) [29, 30]. Ruptures and luxations of the annular ligament in adults have also been described and may be the cause of a painful snapping elbow [34]. The evaluation of the attachments of the annular ligament and its relationship with the other components of the LCL may be important in these cases.

The LCL could be clearly differentiated from the surrounding structures by ultrasound (Fig. 2). The proximal portion of this ligamentous complex was separated from the overlying extensor tendon by a thin echogenic line. This line was made visible in all control subjects and in most of the cadaveric specimens $(80 \%)$ by a dynamic ultrasound evaluation, with gentle angulation of the probe (Fig. 5). Anisotropic artefact, the different orientation of the ligament fibres compared with those of the overlying tendons or even small amounts of loose connective tissue are most likely responsible for the visualisation of this line at ultrasound [15]. At specimen sectioning the LCL and the extensor tendons could be macroscopically differentiated (Fig. 5a).

The differentiation between tendons and ligaments was easier in the normal volunteers. This finding is probably related to the muscular tonus which kept the ligaments and tendons taut, facilitating ultrasound evaluation. Studies have shown that the LCL is taut with the elbow flexed $[35,36]$. There is no consensus on the best position for the ultrasound study of the LCL. Both a $90^{\circ}$ flexion and an extended position have been reported [15, 37]. In our study, for comparison purposes with the anatomical sections, which were obtained with the elbow in extension, we also performed the ultrasound examination of the elbow in this position. The ultrasound evaluation of the LCL in cadavers with the elbow flexed was easier as the ligament fibres are stretched and less prone to anisotropy artefacts. No significant difference was noted with live subjects.

A bony tubercle was identified at the anterior portion of the lateral epicondyle of some of the evaluated lateral epicondyles $(61 \%)$ as a discrete alteration in the epicondyle surface. This structure was identified in the cadaveric sectioning (Fig. 6). This structure was described by Zoner et al. and is thought to mark the attachment site of the extensor carpi radialis brevis tendon [38]. High-definition radiographs of the sectioned specimens demonstrate these findings well. When present, this tubercle helped along with the hyperechogenic line to make the distinction between extensor tendons and the LCL.

The distal attachment of the annular and ulnar components of the LCL on the supinator crest of the ulna has been classified into two different types by Cohen and Hastings [24]. Type I (52\%) morphology is described as a bilobed insertion, with the LUCL inserting distal to the annular ligament. Type II (48\%) morphology is described as a single broad ulnar attachment of the two components. The distal attachments of both the annular ligament and the LUCL were clearly identified in all the volunteers and in 9 out of 10 specimens, allowing the differentiation between those two types of ligamentous insertion (Figs. 3 and $4 b$ ). The clinical significance of identifying these two types of attachment, if any, is yet to be determined. Additionally two ligamentous bands with interposed fatty tissue were recently described at the distal attachment of the annular ligament [3]. With ultrasound the superficial portion of the attachment was identified, and in all the volunteers and in 9 out of 10 specimens hyperechogenic fat tissue could be seen deep to these fibres. However the deep fibres of this ligament could not be separated from the radial head cartilage (Fig. 6).

We hypothesise that the differences in echogenicity of the proximal LCL found between normal volunteers and cadaveric subjects are, at least partially, due to degenerative changes in the ligaments of the cadaveric specimens. Although a histological analysis is necessary to confirm this finding the hypothesis seems plausible when the age difference between the two groups is taken into account.

Limitations of our study include the lack of clinical data in the cadaveric specimens, in particular with regard to previous LCL lesions. Hence, no attempt was made to detect lesions in the specimens evaluated. The age difference of the cadaveric specimens and the normal volunteers should be also acknowledged as a limitation of this study because it is likely to be responsible for some of the differences in the ultrasound aspect of the LCL in between these two groups. Although the ultrasound machines provided high-quality images the fact that they were not the same may be responsible for some of the differences in the ultrasound appearance of the LCL between cadavers and live subjects. Different ultrasound equipment was used for the evaluation of the normal subjects because these examinations were done in a different institution. Further studies are necessary to evaluate the accuracy of ultrasound in detecting alterations in each of these ligaments in a pathological setting.

In conclusion, ultrasound allows the assessment of all the components of the LCL and can distinguish them from overlying tendinous structures. A thorough assessment of the LCL is clinically useful, because each of these components can be injured alone or in association. Knowledge of the ultrasound appearance of the soft tissue and bony structures at the level of the lateral epicondyle is important for the evaluation of lateral elbow abnormalities. 
Open Access This article is distributed under the terms of the Creative Commons Attribution Noncommercial License which permits any noncommercial use, distribution, and reproduction in any medium, provided the original author(s) and source are credited.

\section{References}

1. O’Driscoll SW, Horii E, Bernard F, Morrey BF, Carmichael SW (1992) Anatomy of the ulnar part of the lateral collateral ligament of the elbow. Clin Anat 5:296-303

2. Hannouche D, Bégué T (1999) Functional anatomy of the lateral collateral ligament complex of the elbow. Surg Radiol Anat 21:187-191

3. Sanal HT, Chen L, Haghighi P, Trudell DJ, Resnick DL (2009) Annular ligament of the elbow: MR arthrography appearance with anatomic and histologic correlation. Am J Roentgenol 193:W122W126

4. Mehta JA, Bain GI (2004) Posterolateral rotatory instability of the elbow. J Am Acad Orthop Surg 12:405-415

5. Potter HG, Weiland AJ, Schatz JA, Paletta GA, Hotchkiss RN (1997) Posterolateral rotatory instability of the elbow: usefulness of MR imaging in diagnosis. Radiology 204(1):185-189

6. Mehta JA, Bain GI (2004) Posterolateral rotatory instability of the elbow. J Am Acad Orthop Surg 12:405-415

7. Kijowski R, Smet AAD (2005) Magnetic resonance imaging findings in patients with medial epicondylitis. Skeletal Radiol 34:196-202

8. Maloney MD, Mohr KJ, el Attrache NS (1999) Elbow injuries in the throwing athlete. Difficult diagnoses and surgical complications. Clin Sports Med 18:795-809

9. Kijowski R, Tuite M, Sanford M (2005) Magnetic resonance imaging of the elbow. Part II: abnormalities of the ligaments, tendons, and nerves. Skeletal Radiol 34:1-18

10. Carrino JA, Morrison WB, Zou KH, Steffen RT, Snearly WN, Murray PM (2001) Noncontrast MR imaging and MR arthrography of the ulnar collateral ligament of the elbow: prospective evaluation of two-dimensional pulse sequences for detection of complete tears. Skeletal Radiol 30:625-632

11. Cotten A, Jacobson J, Brossmann J, Pedowitz R, Haghighi P, Trudell D, Resnick D (1997) Collateral ligaments of the elbow: conventional MR imaging and MR arthrography with coronal oblique plane and elbow flexion. Radiology 204:806-812

12. Terada N, Yamada H, Toyama Y (2004) The appearance of the lateral ulnar collateral ligament on magnetic resonance imaging. $\mathrm{J}$ Shoulder Elbow Surg 13:214-216

13. Bianchi S, Martinoli C, Derchi LE, Baert AL, Rizzatto G, Abdelwahad IF, Valle M (2007) Ultrasound of the musculoskeletal system, Springer

14. Zordo TD, Lill SR, Fink C, Feuchtner GM, Jaschke W, BellmannWeiler R, Klauser AS (2009) Real-time sonoelastography of lateral epicondylitis: comparison of findings between patients and healthy volunteers. AJR Am J Roentgenol 193:180-185

15. Connell D, Burke F, Coombes P, McNealy S, Freeman D, Pryde D, Hoy G (2001) Sonographic examination of lateral epicondylitis. AJR Am J Roentgenol 176:777-782

16. Stewart B, Harish S, Oomen G, Wainman B, Popowich T, Moro JK (2009) Sonography of the lateral ulnar collateral ligament of the elbow: study of cadavers and healthy volunteers. AJR Am J Roentgenol 193:1615-1619

17. De Maeseneer M, Jager T, Vanderdood K, Van Roy $P$, Shahabpour M, Marcelis S (2004) Ultrasound during dissection of cadaveric specimens: a new method for obtaining ultrasound-anatomic correlations in musculoskeletal radiology. Eur Radiol 14:870-874
18. McKee MD, Schemitsch EH, Sala MJ, O'driscoll SW (2003) The pathoanatomy of lateral ligamentous disruption in complex elbow instability. J Shoulder Elbow Surg 12:391-396

19. Berg EE, DeHoll D (1999) The lateral elbow ligaments. A correlative radiographic study. Am J Sports Med 27:796-800

20. Greenbaum B, Itamura J, Vangsness CT, Tibone J, Atkinson R (1999) Extensor carpi radialis brevis. An anatomical analysis of its origin. J Bone Joint Surg Br 81:926-929

21. Imatani J, Ogura T, Morito Y, Hashizume H, Inoue H (1999) Anatomic and histologic studies of lateral collateral ligament complex of the elbow joint. J Shoulder Elbow Surg 8:625-627

22. Beckett KS, McConnell P, Lagopoulos M, Newman RJ (2000) Variations in the normal anatomy of the collateral ligaments of the human elbow joint. J Anat 197(Pt 3):507-511

23. Olsen BS, Søjbjerg JO, Dalstra M, Sneppen O (1996) Kinematics of the lateral ligamentous constraints of the elbow joint. J Shoulder Elbow Surg 5:333-341

24. Cohen MS, Hastings H (1997) Rotatory instability of the elbow. The anatomy and role of the lateral stabilizers. J Bone Joint Surg Am 79:225-233

25. Seki A, Olsen BS, Jensen SL, Eygendaal D, Søjbjerg JO (2002) Functional anatomy of the lateral collateral ligament complex of the elbow: configuration of Y and its role. J Shoulder Elbow Surg 11:53-59

26. Dunning CE, Zarzour ZD, Patterson SD, Johnson JA, King GJ (2001) Ligamentous stabilizers against posterolateral rotatory instability of the elbow. J Bone Joint Surg Am 83-A:1823-1828

27. McAdams TR, Masters GW, Srivastava S (2005) The effect of arthroscopic sectioning of the lateral ligament complex of the elbow on posterolateral rotatory stability. J Shoulder Elbow Surg 14:298-301

28. Charalambous CP, Stanley JK, Siddique I, Aster A, Gagey O (2009) Posterolateral rotatory laxity following surgery to the head of the radius: biomechanical comparison of two surgical approaches. J Bone Joint Surg Br 91:82-87

29. Kim MC, Eckhardt BP, Craig C, Kuhns LR (2004) Ultrasonography of the annular ligament partial tear and recurrent "pulled elbow". Pediatr Radiol 34:999-1004

30. Kosuwon W, Mahaisavariya B, Saengnipanthkul S, Laupattarakasem W, Jirawipoolwon P (1993) Ultrasonography of pulled elbow. J Bone Joint Surg Br 75:421-422

31. De Smet AA, Winter TC, Best TM, Bernhardt DT (2002) Dynamic sonography with valgus stress to assess elbow ulnar collateral ligament injury in baseball pitchers. Skeletal Radiol 31:671-676

32. Finlay K, Ferri M, Friedman L (2004) Ultrasound of the elbow. Skeletal Radiol 33:63-79

33. Bredella MA, Tirman PF, Fritz RC, Feller JF, Wischer TK, Genant HK (1999) MR imaging findings of lateral ulnar collateral ligament abnormalities in patients with lateral epicondylitis. AJR Am J Roentgenol 173:1379-1382

34. Huang G, Lee C, Lee H, Chen C (2005) MRI, arthroscopy, and histologic observations of an annular ligament causing painful snapping of the elbow joint. AJR Am J Roentgenol 185:397-399

35. Moritomo $\mathrm{H}$, Murase $\mathrm{T}$, Arimitsu S, Oka K, Yoshikawa H, Sugamoto K (2007) The in vivo isometric point of the lateral ligament of the elbow. J Bone Joint Surg Am 89:2011-2017

36. Armstrong AD, Ferreira LM, Dunning CE, Johnson JA, King GJW (2004) The medial collateral ligament of the elbow is not isometric: an in vitro biomechanical study. Am J Sports Med 32:85-90

37. Martinoli C, Bianchi S, Giovagnorio F, Pugliese F (2001) Ultrasound of the elbow. Skeletal Radiol 30:605-614

38. Zoner CS, Buck FM, Cardoso FN, Gheno R, Trudell DJ, Randall TD, Resnick D (2010) Detailed MRI-anatomic study of the lateral epicondyle of the elbow and its tendinous and ligamentous attachments in cadavers. AJR Am J Roentgenol 195:629-636 